\title{
Cell cycle-specific changes in hTERT promoter activity in normal and cancerous cells in adenoviral gene therapy: A promising implication of telomerase-dependent targeted cancer gene therapy
}

\author{
YOSHITERU MUROFUSHI ${ }^{1 *}$, SATOSHI NAGANO ${ }^{1,4^{*}}$, JUNICHI KAMIZONO $^{1,4}$, TOMOYUKI TAKAHASHI $^{1,2}$, \\ HISAYOSHI FUJIWARA ${ }^{5}$, SETSURO KOMIYA ${ }^{4}$, TOYOJIRO MATSUISHI ${ }^{1,3}$ and KEN-ICHIRO KOSAI ${ }^{1,3}$ \\ ${ }^{1}$ Division of Gene Therapy and Regenerative Medicine, Cognitive and Molecular Research Institute of Brain Diseases, \\ Kurume University, Departments of ${ }^{2}$ Advanced Therapeutics and Regenerative Medicine and ${ }^{3}$ Pediatrics and Child Health, \\ Kurume University School of Medicine, 67 Asahi-machi, Kurume 830-0011; ${ }^{4}$ Department of Orthopaedic Surgery, \\ Graduate School of Medical and Dental Sciences, Kagoshima University, 8-35-1 Sakuragaoka, Kagoshima 890-8520; \\ ${ }^{5}$ Department of Cardiology, Respiratory and Nephrology, Regeneration \& Advanced Medical Science, \\ Graduate School of Medicine, Gifu University, 1-1 Yanagido, Gifu 501-1194, Japan
}

Received February 28, 2006; Accepted April 13, 2006

\begin{abstract}
Based on the finding that telomerase is reactivated solely in cancer cells, the human telomerase reverse transcriptase (hTERT) promoter has recently been used to target cancer cells by gene therapy. The recent, surprising observation that telomerase is physiologically activated even in normal somatic cells during $\mathrm{S}$-phase has raised concerns as to the safety of this methodology. To clarify this issue, the present study carefully examined the changes in endogenous telomerase activities, hTERT mRNA expression, and hTERT promoter-based transgene expression in normal and cancer cells at synchronized phases of the cell cycle. Telomerase activity and hTERT expression were detected at variable, but relatively high, levels in all 12 cancer cell lines, while both were undetectable in the 11 normal cell lines. In HepG2
\end{abstract}

Correspondence to: Dr Ken-ichiro Kosai, Division of Gene Therapy and Regenerative Medicine, Cognitive and Molecular Research Institute of Brain Diseases, Kurume University, 67 Asahimachi, Kurume 830-0011, Japan

E-mail: kosai@med.kurume-u.ac.jp

${ }^{*}$ Contributed equally

Abbreviations: hTERT, human telomerase reverse transcriptase; CRA, conditionally replicating adenovirus; HPRT, hypoxanthine guanine phosphoribosyl transferase; TRAP, telomeric repeat amplification protocol; RSV, Rous sarcoma virus long-terminal repeat; CMV promoter, cytomegalovirus immediate-early gene enhancer/promoter; MOI, multiplicity of infection

Key words: adenovirus, cancer gene therapy, cancer-specificity, hTERT, targeting cancer, promoter, telomerase, telomere cancer cells, the highest levels of hTERT expression and telomerase activity, seen in the $\mathrm{G}_{1} / \mathrm{S}$ - and S-phases, were 2-3-fold higher than the lowest levels of both, observed in $\mathrm{G}_{0}$-phase and during asynchronization. No hTERT expression or telomerase activitiy could be detected in normal WI-38 fibroblasts at any phase of the cell cycle, including S-phase. Consequently, activity of the shorter hTERT promoter, which was transferred into HepG2 cancer cells via adenovirus transduction, was stronger than that of the longer hTERT promoter at all phases and that of two representatives of ubiquitously strong promoters, at both S-phase and asynchronization, but not at $\mathrm{G}_{0}$-phase. In contrast, neither of hTERT promoters induced detectable transgene expressions in normal WI-38 cells at any cell cycle phase, including S-phase. These results, particularly the lack of problematic levels of S-phase-specific activation of hTERT promoters in normal cells, have promising implications for hTERT promoter-based targeted gene therapy of cancer.

\section{Introduction}

Telomeres are the distal ends of human chromosomes composed of tandem repeats of the sequence TTAGGG. These sites may function to stabilize chromosomal ends and prevent chromosome degradation, end-to-end fusion, rearrangement, and loss (1-4). Telomeres in somatic cells undergo progressive shortening with each successive cell division; it has been hypothesized that the reduction in telomere length may function as an intrinsic clock involved in the onset of cellular senescence $(1,2,5)$. In immortal cells, telomeres are resynthesized and maintained by telomerase, a specialized DNA polymerase responsible for replication of chromosomal ends $(6,7)$. Human telomerase reverse transcriptase (hTERT), the catalytic subunit of human telomerase, is the major determinant of telomerase activity; ectopic expression of hTERT is sufficient to reconstitute telomerase activity in telomerase-negative cells 
$(1,2,8,9)$. Human telomerase activity and hTERT expression are detected in the majority $(>90 \%)$ of human cancer cells, but are typically absent from normal cells $(1,2,10,11)$. In conjunction with the fact that immortalization correlates well with a stabilization of telomere length, it has been proposed that human cancer cells achieve immortalization through illegitimate activation of telomerase expression $(1,2,6)$.

One of the major challenges of cancer gene therapy is the restriction of transgene expression to cancer cells, because non-specific, extratumoral expression of therapeutic genes would result in the destruction of normal tissues $(12,13)$. Tissue-specific promoters, such as the carcinoembryonic antigen promoter (14), have been used as a treatment for adenocarcinoma to achieve tumor-specific transgene expression. These tissue-specific promoters, however, have the disadvantage of targeting only limited cancer types. In addition, they may exhibit insufficient cancer specificity (leaky activation in normal cells) and/or weak activity, even in cancer cells. To increase the potential efficacy of gene therapy, hTERT promoters have been utilized for cancer gene therapy $(13,15,16)$. Although the lengths of the promoter used differed between reports, hTERT promoter-based transgene regulation should be able to target a broad range of cancers with little effect on mature somatic cells. Recently, the hTERT promoter was also used to generate a conditionally replicating adenovirus (CRA); several studies have demonstrated that hTERT promoter-based CRA can selectively replicate in and kill a panel of cancer cells (17-20).

The majority of previous studies examining hTERT promoter-based cancer gene therapy focused on achieving efficacy in particular cancer models $(13,15,16,21,22)$. The potential adverse effects in normal cells have not yet been thoroughly investigated, although some concerns have been implicated by the fact that telomerase activity is observed at low levels in certain normal cells, such as bone marrow and peripheral blood mononuclear cells $(23,24)$. Relatively high levels of telomerase activity have been reported in hematopoietic cells, the basal layer of the epidermis, endometrial tissues during the menstrual cycle, fetal tissues, and the proliferative zone of intestinal crypts $(1,24,25)$. Surprisingly and unexpectedly, endogenous telomerase activity and hTERT expression could also be detected in an S-phase-specific manner in common normal somatic fibroblasts, which were previously thought to lack both; the upregulated telomerase played a physiological role in the proliferation of normal cells (26). These results strongly suggest a need to evaluate carefully the potential adverse effects of such treatment, specifically determining if leaky expression of a transgene under the control of the hTERT promoter would occur in normal cycling cells to achieve harmful levels. This study carefully examined the changes in telomerase activity, hTERT expression, and hTERT promoter-based transgene expression in normal and cancer cells at specific phases of the cell cycle. The obtained results provide important general implications for hTERT promoter-based targeted cancer gene therapy.

\section{Materials and methods}

Cell culture. The human cancer cell lines MKN-1, -28, and -45 (gastric cancer), HCT-15, LoVo, and colo-205 (colon cancer),
HepG2 and Hep3B (hepatoma), HeLa (cervical cancer), and HOS-MNNG, KHOS-NP, and SaOS-2 (osteosarcoma) were obtained and maintained as described previously (20). Normal human cell lines, WI-38 and IMR-90 (lung fibroblasts), were obtained from the RIKEN Cell Bank (Tsukuba, Japan), while MRC-5 (lung fibroblasts) and HUV-EC (human umbilical vein endothelial cells) were obtained from the Health Science Research Resources Bank (Osaka, Japan). Primary cultured human cells, NHDF (dermal fibroblasts), NHOst (osteoblasts), HMVEC-d (dermal-derived microvascular endothelial cells), HMEC (mammary epithelial cells), PrEC (prostate epithelial cells), HRE (renal epithelial cells), and SAEC (small airway epithelial cells) were obtained from Cambrex Bio Science Walkersville (Walkersville, MD, USA). All normal cells were maintained according to the manufacturer's protocol.

Reverse transcription-polymerase chain reaction analysis $(R T-P C R)$. Extraction of total RNA from cells and semiquantitative RT-PCR analysis of hTERT and hypoxanthine guanine phosphoribosyl transferase (HPRT) mRNA levels were performed as described previously $(27,28)$. For nested RT-PCR analysis, cDNA was subjected to initial PCR amplification with 20 cycles of $94^{\circ} \mathrm{C}$ for $30 \mathrm{sec}, 59^{\circ} \mathrm{C}$ for $60 \mathrm{sec}$ and $74^{\circ} \mathrm{C}$ for $60 \mathrm{sec}$ in the presence of Taq DNA polymerase (Promega, Madison, WI, USA) and primer set 1 (P1; sense, 5'-TTCCTGCACTGGCTGATGAGTGT-3', and antisense, 5'-CGCTCGGCCCTCTTTTCTCTG-3') (29). Subsequently, $1 / 25$ of the amplified cDNA was subjected to a second PCR amplification of 35 cycles of $94^{\circ} \mathrm{C}$ for $45 \mathrm{sec}$, $60^{\circ} \mathrm{C}$ for $45 \mathrm{sec}$ and $72^{\circ} \mathrm{C}$ for $90 \mathrm{sec}$ with Taq DNA polymerase and primer set 2 (P2; sense, 5'-CCTGCTGGATTACATTAA AGCACTG-3', and antisense, 5'-AAGGGCATATCCAACA ACAA-3') (7).

Endogenous telomerase activity. Endogenous telomerase activity in cells was examined using the telomeric repeat amplification protocol (TRAP) with Telo TAGGG Telomerase PCR ELISA ${ }^{\text {PLUS }}$ kit (Roche Diagnostics GmbH, Mannheim, Germany) according to the manufacturer's protocol.

Cell cycle analysis. Cell cycle synchronization was induced as described (30), with the following modifications. Briefly, synchronization of cells in $\mathrm{G}_{0^{-}}$, S-, or $\mathrm{G}_{2} / \mathrm{M}$-phases was achieved by treatment with serum starvation $(0.5 \%)$ for $72 \mathrm{~h}$, $0.3 \mathrm{mM}$ hydroxyurea (Sigma-Aldrich, St. Louis, MO, USA) for $32 \mathrm{~h}$, or $0.4 \mu \mathrm{g} / \mathrm{ml}$ nocodazole (Sigma-Aldrich) for $20 \mathrm{~h}$, respectively. Cells were synchronized in $G_{1} / S$ phase by treatment with $5 \mathrm{mM}$ thymidine (Sigma-Aldrich) for $20 \mathrm{~h}$, followed by treatment with $5 \mu \mathrm{g} / \mathrm{ml}$ aphidicolin (SigmaAldrich) for $16 \mathrm{~h}$ (30). The percentage of cells entering each phase of the cell cycle was determined by flow cytometric analysis of propidium iodide-stained cells using a FACSCalibur cytometer (Becton Dickinson, San Jose, CA, USA) and ModFit software (Verity, Topsham, ME, USA).

Generation of adenoviral vectors. The short (260-bp; -181 to +79) and long (1454-bp; -1375 to +79) hTERT promoters [hTERT(S) and hTERT(L)] were isolated by $M l u \mathrm{I} / B g l \mathrm{II}$ digestion from the pGL3-181 and pGL3-1375 plasmids (the kind gift of Dr S. Kyo, Kanazawa University, Kanazawa, 
A

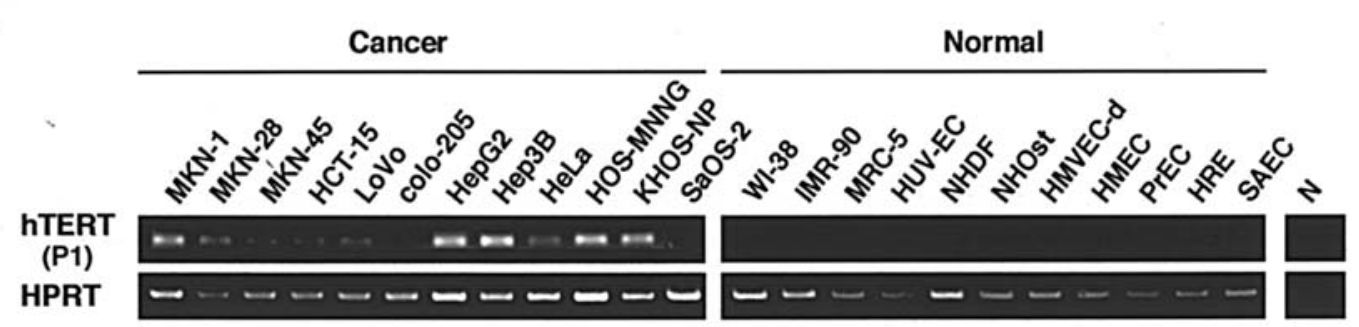

B

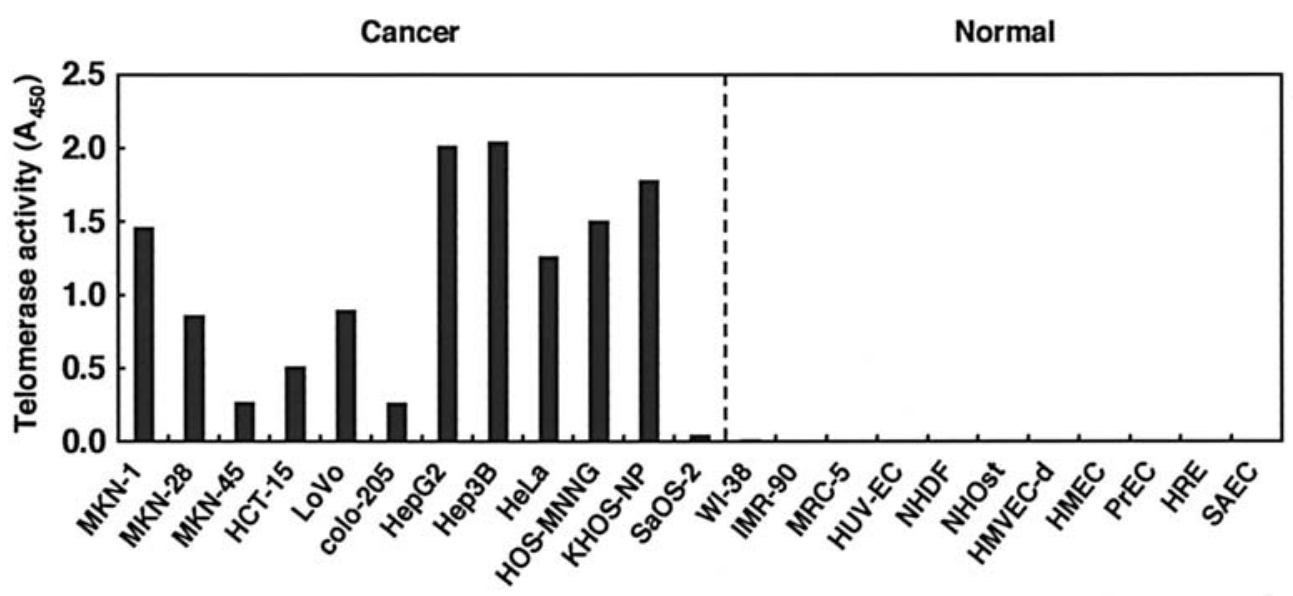

Figure 1. Endogenous hTERT expression and telomerase activity in a variety of cancer and normal cells. (A) Endogenous hTERT mRNA was detected by standard RT-PCR using the primer set 1 (P1) in all of 12 cancer cell lines, but was undetectable in 11 normal cells. Amplification of the HPRT gene served as an internal control. N, no template served as a negative control. (B) Endogenous telomerase activity was detected by TRAP assay at varying levels in all cancer cell lines, but could not be detected in any of the normal cells. Data are represented as the levels at $\mathrm{A}_{450}$.

Japan), respectively (31). We generated E1-deleted replication-defective adenoviral vectors, Ad.hTERT(S)-LacZ, Ad.hTERT(L)-LacZ, Ad.RSV-LacZ, and Ad.CMV-LacZ, which express the LacZ gene under the control of the hTERT(S) promoter, the hTERT(L) promoter, the Rous sarcoma virus long-term repeat (RSV) promoter, and the cytomegalovirus immediate-early gene enhancer/promoter (CMV promoter), respectively $(20,32)$. Adenoviral stocks were prepared and titered as described previously $(33,34)$.

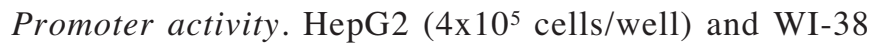
$\left(1.2 \times 10^{5}\right.$ cells/well) cells in 6 -well plates were infected with each adenoviral vector at a multiplicity of infection (MOI) of 10 for $1 \mathrm{~h}$ and at MOI of 30 for $24 \mathrm{~h}$, respectively, conditions which provided almost similar gene transduction efficiencies in both cell types (about 30\%) without apparent cytotoxicity. The cells were synchronized at each phase of the cell cycle as described above and subsequently harvested. $\beta$-galactosidase activity was measured using a $\beta$-Galactosidase Enzyme Assay System (Promega) as described previously $(14,20)$.

\section{Results}

Endogenous hTERT mRNA levels and telomerase activities in various human cancerous and normal cell types. Consistent with our previous results, hTERT mRNA expression was readily detected by standard semi-quantitative RT-PCR analysis in all of the examined cancer cells, which were derived from a variety of tissue origins; a more sensitive nested RT-PCR was not necessary for detection. The expression levels varied considerably among cell types (Fig. 1A); notably, both hepatoma cell lines (HepG2 and Hep3B) and two of the three osteosarcoma cell lines (HOS-MNNG and KHOS-NP) exhibited high expression levels of hTERT mRNA. Endogenous telomerase activity was detected by TRAP assays in all 12 cancer cell lines at varying levels; eleven cancer cell lines exhibited relatively high activity, while SaOS-2 cells, which have been reported as a telomerase-negative osteosarcoma $(35,36)$, displayed only low levels of telomerase activity (Fig. 1B). The telomerase activity in each cancer cell line correlated well with the expression levels of hTERT mRNA. In contrast, neither hTERT mRNA expression nor telomerase activity could be detected in the 11 normal cell lines derived from a variety of tissues.

Thus, these findings support the widely accepted notion that hTERT is the telomerase catalytic subunit and is reactivated specifically in cancer cells $(6,7,37)$, although the diversity of levels between individual cell lines is relatively large.

Expressions of endogenous hTERT $m R N A$ in cancer and normal cells at each phase of cell cycle. Previous studies demonstrated that telomerase activity in cancer cells changed throughout the cell cycle $(30,38)$. A recent study revealed that hTERT was also expressed in normal human fibroblasts, which were previously thought to lack hTERT expression and telomerase activity. This expression, however, was restricted to the S-phase (26). These results suggest that hTERT 

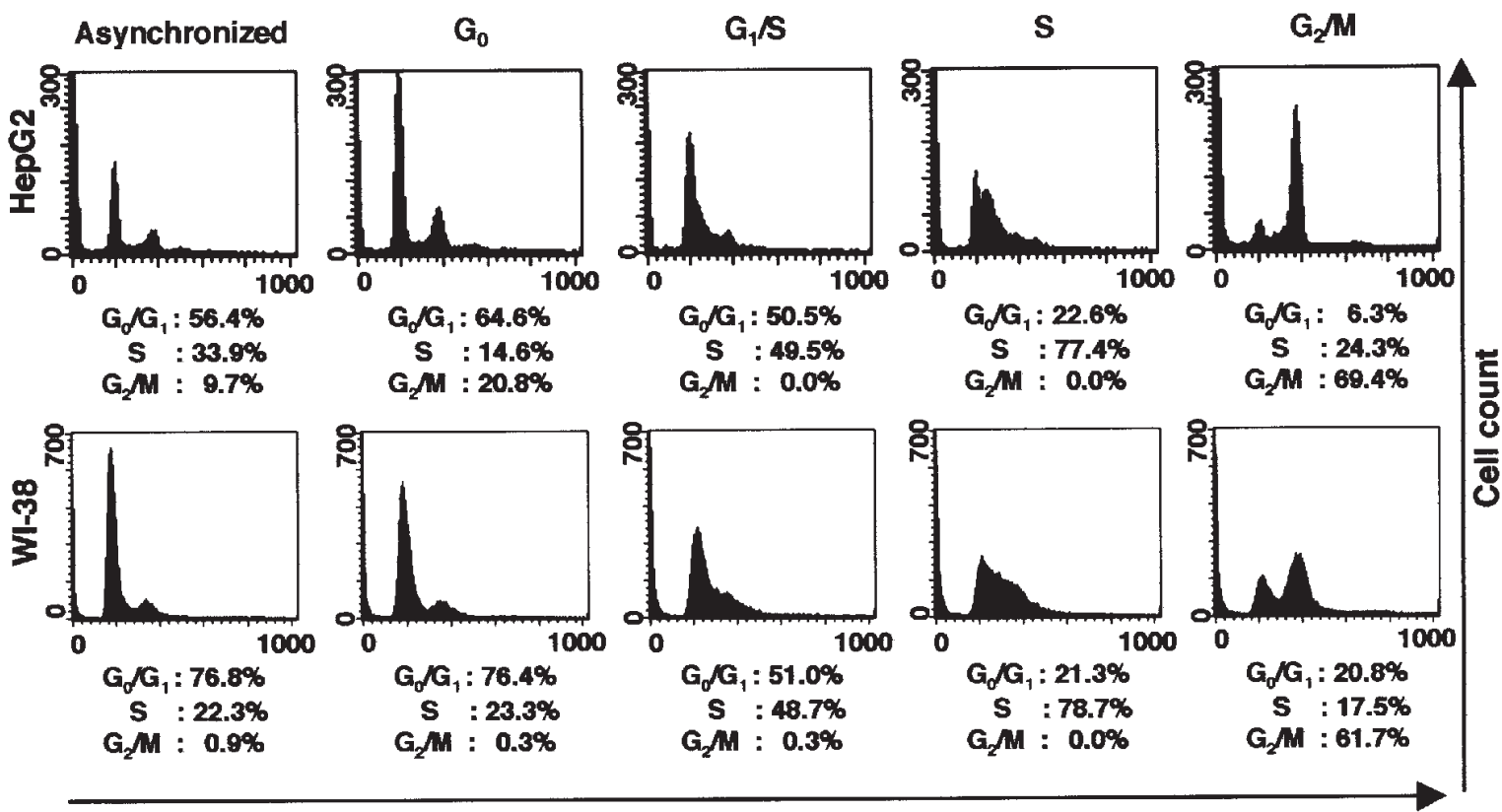

DNA content

Figure 2. Synchronization of cancer and normal cells at specific phases of the cell cycle. HepG2 cancer and WI-38 normal cells were synchronized at $\mathrm{G}_{0^{-}}$, $\mathrm{G}_{1} / \mathrm{S}-, \mathrm{S}-$, and $\mathrm{G}_{2} / \mathrm{M}$-phases by treatment with $0.5 \%$ serum starvation for $72 \mathrm{~h}, 5 \mathrm{mM}$ thymidine for $20 \mathrm{~h}$ with $5 \mu \mathrm{g} / \mathrm{ml}$ aphidicolin for additional $16 \mathrm{~h}, 0.3 \mathrm{mM}$ hydroxyurea for $32 \mathrm{~h}$, and $0.4 \mu \mathrm{g} / \mathrm{ml}$ nocodazole for $20 \mathrm{~h}$, respectively. The percentage of the cells entering the specific phase of the cell cycle was determined by flow cytometric analysis of propidium iodide-stained cells.

expression and/or telomerase activity might be detected in many normal cell types during S-phase of the cell cycle. We next compared the maximal levels of both hTERT expression and telomerase activity in normal cells with those in cancer cells. After synchronizing HepG2 hepatoma cells and WI-38 normal fibroblasts, we examined hTERT mRNA expression and telomerase activity. We chose the HepG2 and WI-38 cell lines for these experiments, because they have been widely used for both telomerase $(26,30,37)$ and adenoviral gene therapy studies $(20,34,39)$.

Through several pilot studies, we determined the optimal conditions for cell synchronization and adenoviral gene delivery/expression, as shown in Materials and methods. HepG2 and WI-38 cells were successfully synchronized and/or arrested at $\mathrm{G}_{0^{-}}, \mathrm{G}_{1} / \mathrm{S}-\mathrm{S}, \mathrm{S}-$, or $\mathrm{G}_{2} / \mathrm{M}$-phases by treatment with serum starvation, thymidine/aphidicolin, hydroxyurea, or nocodazole, respectively. Assessment of DNA content by flow cytometry demonstrated that the percentages of cells in the desired phases of the cell cycle were high and similar between HepG2 and WI-38 cells (Fig. 2).

We examined the expression of endogenous hTERT mRNA in HepG2 and WI-38 cells by RT-PCR analysis at each phase of the cell cycle. HepG2 cancer cells exhibited high levels of hTERT mRNA at all phases of the cell cycle, including $\mathrm{G}_{0}$-phase, as well as under conditions of asynchronization (Fig. 3A). In contrast, hTERT mRNA expression could not be detected in WI-38 normal fibroblasts at any phase of cell cycle, either by standard RT-PCR analysis or the more sensitive nested RT-PCR analysis (Fig. 3B), although a recent report noted that hTERT mRNA could be detected in WI-38 cells during S-phase by standard PCR using the same primers (P2) (26).
A

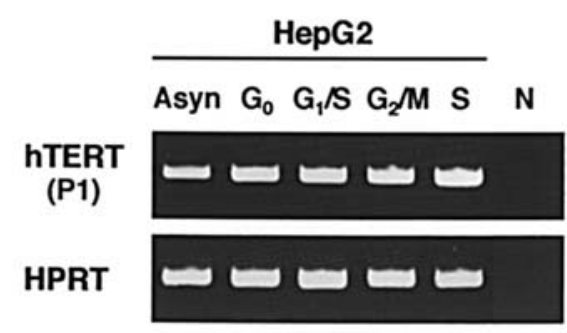

B

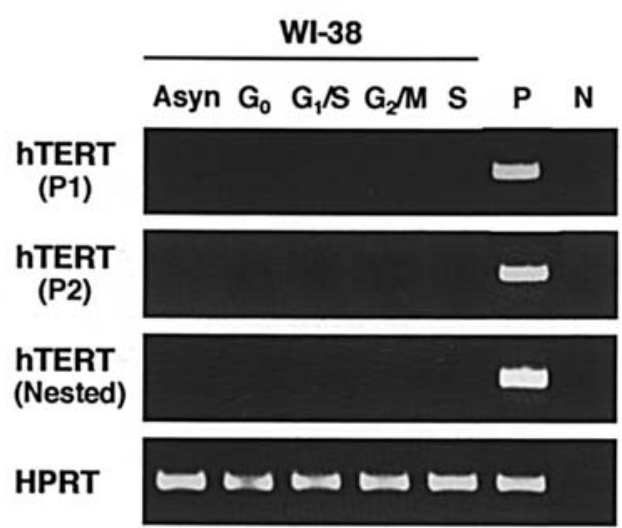

Figure 3. Endogenous hTERT mRNA expression at specific phases of the cell cycle in cancer and normal cells. HepG2 (A) and WI-38 (B) cells were synchronized at each phase of cell cycle, as described in Fig. 2. hTERT mRNA expression was then examined by RT-PCR. (A) hTERT mRNA expression was prominent in $\mathrm{HepG} 2$ cancer cells at all phases by standard RT-PCR analysis using the primer set 1 (P1). (B) In contrast, hTERT mRNA expression could not be detected by either standard RT-PCR using primer sets 1 or 2 (P2) or by nested RT-PCR. The HPRT gene was amplified as an internal control. Asyn, asynchronized cells; N, no template served as a negative control; P, template from HepG2 served as a positive control. 


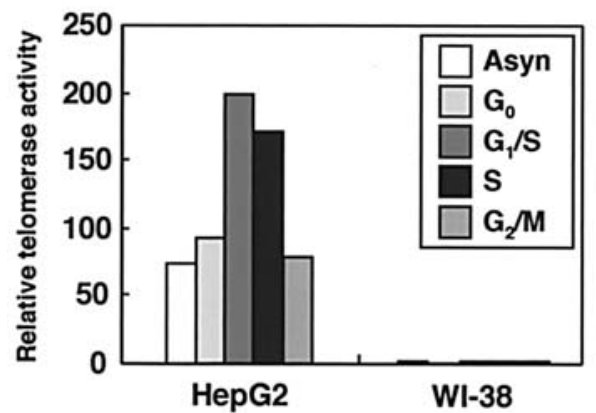

Figure 4. Endogenous telomerase activities at specific phases of the cell cycle in cancerous and normal cells. HepG2 and WI-38 cells were synchronized at each phase of the cell cycle, as described in Fig. 2. Telomerase activities were examined by TRAP assay. Data were represented as telomerase activity normalized to the supplied standard control.

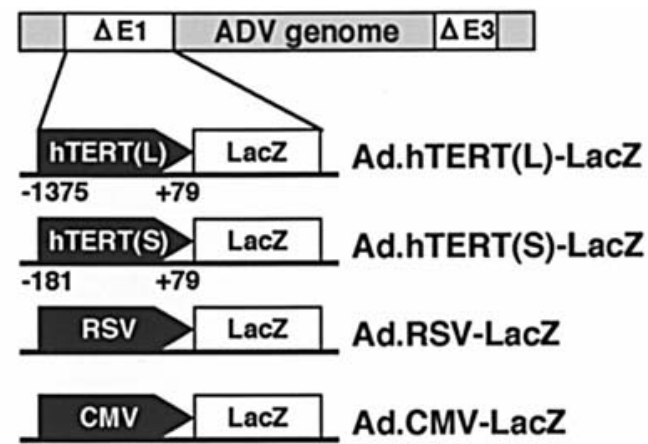

Figure 5. Schematic representation of replication-defective adenoviral vectors. A LacZ gene, downstream of the hTERT(S), hTERT(L), RSV, or CMV promoters was inserted into E1-deleted replication-defective adenoviral vectors.

Endogenous telomerase activities in cancer and normal cells at each phase of cell cycle. Next, we examined endogenous telomerase activity in HepG2 and WI-38 cells by TRAP assay at each phase of cell cycle. High levels of telomerase activity were observed in HepG2 hepatoma cells throughout the cell cycle, including in $\mathrm{G}_{0}$-phase, although cell cycle-dependent changes of telomerase activity levels were prominent (Fig. 4). Telomerase activity at $\mathrm{G}_{1} / \mathrm{S}$ - and $\mathrm{S}$-phases were 2-3-fold higher than that seen under conditions of asynchronization. Despite the S-phase-specific upregulation $(30,38)$, the fact that telomerase activities in $G_{0}$ and $G_{2} / M$ (i.e., out of $S$-phase of cell cycle) remain relatively high is surprising. In contrast, no telomerase activity was detected in WI-38 normal fibroblasts at any phase of the cell cycle. These results suggest that both baseline hTERT expression and the resulting telomerase activity are significantly higher in cancer cells than the maximal levels seen in normal cells, despite the tight regulation of hTERT expression in both cell types in a cell cycle-dependent manner.

Cell cycle-dependent transgene regulation in cancer and normal cells using the hTERT promoters carried in an adenoviral vector. Although several studies have identified hTERT promoters of different lengths, a recent report demonstrated that two hTERT promoters [hTERT(L): -1375 to +79 and hTERT(S): -181 to +79 ] had similar, potent activities in several cancer cell lines (31). We therefore constructed four E1-deleted replication-defective adenoviral vectors, Ad.hTERT(L)-LacZ, Ad.hTERT(S)-LacZ, Ad.RSV-LacZ, and Ad.CMV-LacZ, which expressed LacZ under the control of the hTERT(L), hTERT(S), RSV, and CMV promoters; the latter two, both strong promoters functioning in all cell types, served as positive controls (Fig. 5). We initially infected either HepG2 or WI-38 cells with each adenoviral vector. After either synchronizing the cells at $\mathrm{G}_{0^{-}}$or $\mathrm{S}$-phase or leaving the cells without any synchronization, we measured $\beta$-galactosidase activity, as described in the Materials and methods.

Highly S-phase-specific transgene expression was observed in HepG2 cancer cells after adenoviral delivery of transgenes controlled by either hTERT promoter; the activities of both hTERT promoters in HepG2 cells at S-phase were 5-7-fold higher than those seen at $\mathrm{G}_{0}$-phase (Fig. 6). Unexpectedly, the activity of hTERT(S) was significantly higher than that of
HepG2

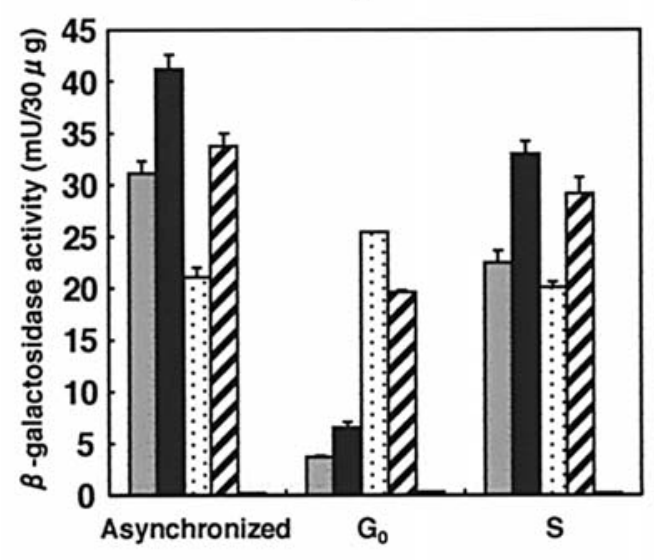

WI-38

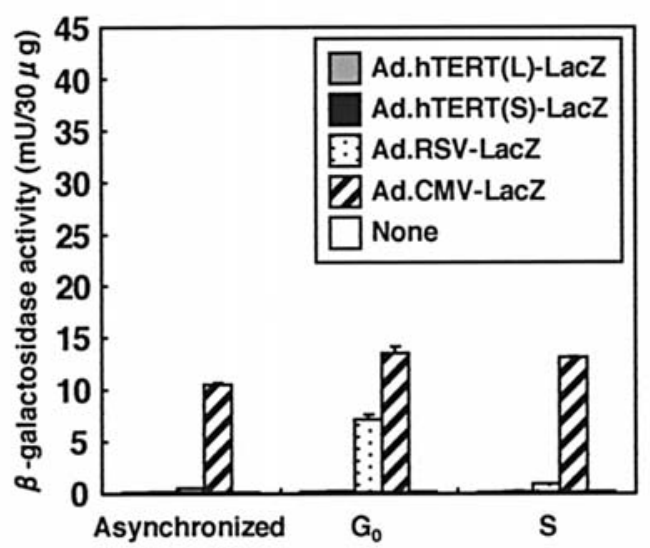

Figure 6. hTERT promoter activity at specific phases of the cell cycle in cancerous and normal cells. HepG2 and WI-38 cells were infected with either adenoviral vector shown in Fig. 5, then synchronized at each phase of the cell cycle as described in Fig. 3. We then measured B-galactosidase activities; each bar represents the mean \pm the standard error. 
hTERT(L) in HepG2 cells under all conditions, including $\mathrm{G}_{0^{-}}$and S-phases. Notably, hTERT(S) activity at S-phase and under conditions of asynchronization, but not during $\mathrm{G}_{0}$-phase, was higher than RSV and CMV promoter activities. In contrast, no hTERT(S) or hTERT(L) activity could be detected in WI-38 normal fibroblasts at any phase of the cell cycle or under conditions of asynchronization.

\section{Discussion}

Although the anti-cancer effects and cancer-selectivity of hTERT promoter-based cancer gene therapy look promising $(13,15,16,21,22)$, the potential safety issues have remained concerning, because of both the potential telomerase activity in subsets of normal cells and the physiological upregulation of telomerase activity specifically during S-phase of the cell cycle, recently reported even in normal somatic cells, which were previously believed to lack both hTERT expression and telomerase activity (1,23-25). Through careful analysis of telomerase throughout the cell cycle, we elucidated the critical potential safety issues of hTERT promoter-based gene therapy. The results were promising, supporting the safe clinical applicability of hTERT promoter-based gene therapy. We observed high cancer-specificity of endogenous telomerase activity and hTERT expression (Fig. 1); the generality of this finding was verified using 12 cancerous and 11 normal cell types, including WI-38 normal fibroblasts previously used by Masutomi et al (26). In addition, endogenous hTERT expression, endogenous telomerase activity, and hTERT promoter activity in WI-38 normal fibroblasts were undetectable at all phases of the cell cycle, including S-phase. This result sharply contrasted the high levels of all three seen in HepG2 cancer cells even at $\mathrm{G}_{0}$-phase. The discrepancy of our results with the previous study reporting detectable telomerase activity in normal cells may result from the different experimental systems and methods used; these methods included the release after synchronization in contrast to direct synchronization to obtain specific phases of the cell cycle, conventional transfection in contrast to adenoviral gene transduction, and the sensitive immunoprecipitation-TRAP as opposed to the standard TRAP assay in the previous and current studies, respectively (26).

The recently-proposed theory that S-phase-specific activation of telomerase is physiologically necessary for proliferation, but not the maintenance of telomere length, of normal cells remains a possibility (26). The levels of telomerase activity in normal cells, however, are significantly lower than those in cancer cells, being almost undetectable by the standard TRAP assay. The abnormally high telomerase activities in cancer cells may play a different, pathological role in the maintenance of the telomere length, leading to cancer cell immortalization $(1,2,6)$. The differences in hTERT expression levels and hTERT promoter activities between cancer and normal cells should be much larger than the fluctuations seen in each cell subset throughout the cell cycle, regardless of parent cell type. Consequently, leaky transgene expression in normal cells remains close to undetectable levels, which likely does not approach a problematic level for the majority of hTERT promoter-based targeting cancer gene therapies.
Insufficient activity of tissue-specific promoters, as well as insufficient cancer-specificity has remained the critical problem with tissue-specific promoter-based targeting cancer gene therapy (40-42). These drawbacks significantly diminish the clinical utility of this technique, as weak anti-cancer effects often result in little or no benefit. The activity of hTERT(S) promoters were stronger than that of the RSV and CMV promoters, representative strong promoters, in HepG2 cancer cells under conditions of asynchronization and during S-phase, although it was weaker than the RSV and CMV promoters at $\mathrm{G}_{0}$-phase. While stronger promoter activity than that of RSV and CMV does not guarantee an increased efficacy in all cancer gene therapy strategies, previous studies suggested that stronger therapeutic gene expression resulted in more beneficial outcomes using a variety of cancer gene therapy approaches $(12,13)$. In previous studies, the RSV promoter was the best of three representative strong promoters for achieving optimal therapeutic expression levels of a suicide gene, providing maximal anti-cancer effects without conspicuous adverse side effects in the treatment of metastatic liver cancer (14). Therefore, a good quality for a useful cancerspecific promoter should be stronger activity than the RSV promoter in targeted cancer cells (14). Thus, stronger activity of the hTERT(S) promoter than those of RSV and CMV promoters is clearly beneficial, supporting the usefulness of hTERT(S) in cancer gene therapy. It would be interesting to compare hTERT(S) activity with therapeutic potential using specific therapeutic genes and cancer models in future studies that focus on potential clinical applications.

Previous studies have utilized several different hTERT promoter lengths of 1720-bp $(-1543$ to +77$)(43,44), 457$-bp $(-378$ to +79$)(13,22), 204-b p(-239$ to -36$)(16,17)$, and 260-bp $(-181$ to +79$)(15,21)$ for gene therapy strategies. Each hTERT promoter region worked well when examined individually, but these have not been compared with each other in gene therapy strategies. We therefore carefully evaluated the activities and cancer specificities of the longest [hTERT(L); 1375 to +79 ] and the shortest [hTERT(S); -181 to +79] hTERT promoters in specific phases of the cell cycle. These studies elucidated that hTERT(S) exerted stronger activity in cancer cells at all phases of the cell cycle and under conditions of asynchronization than the hTERT(L) promoter. The differential promoter activities may be explained by negative regulatory elements between -578 and -378 , upstream of the transcriptional start site of the hTERT gene; a previous study suggested that MZF-2 (myeloid-specific zinc finger protein 2) bound to this site, potentially playing a role in the transcriptional repression of hTERT (45). The most important finding, however, for the application of gene therapy technology to clinical medicine is that neither hTERT(L) nor hTERT(S) exhibited any detectable promoter activity in normal cells at any phase of the cell cycle, including S-phase. Taken together, this study indicates that hTERT(S) will be effective and safe for future targeted cancer gene therapy, at least in combination with adenoviral gene therapy.

In conclusion, hTERT(S), the suitable hTERT promoter, carried by an adenoviral vector conferred strong transgene expression in a strictly cancer- and S-phase-specific manner. The levels of S-phase-specific hTERT promoter activity in 
normal cells were virtually undetectable, which will likely not be problematic for targeted cancer gene therapy.

\section{Acknowledgments}

This study was supported in part by a Health and Labour Sciences Research Grants for Third Term Comprehensive Control Research for Cancer from the Ministry of Health, Labour, and Welfare, Japan. We thank Maiko Saito and Aya Niihara for their technical assistance, Dr Satoru Kyo for providing valuable materials and Dr Yoshiro Koda (Kurume University) for technical advice and help.

\section{References}

1. Keith WN, Bilsland A, Evans TR and Glasspool RM: Telomerase-directed molecular therapeutics. Expert Rev Mol Med 2002: 1-25, 2002

2. Liu L, Lai S, Andrews LG and Tollefsbol TO: Genetic and epigenetic modulation of telomerase activity in development and disease. Gene 340: 1-10, 2004.

3. Blackburn EH: Structure and function of telomeres. Nature 350: $569-573,1991$.

4. Greider CW: Chromosome first aid. Cell 67: 645-647, 1991.

5. Harley CB, Futcher AB and Greider CW: Telomeres shorten during ageing of human fibroblasts. Nature 345: 458-460, 1990.

6. Meyerson M, Counter CM, Eaton EN, et al: hEST2, the putative human telomerase catalytic subunit gene, is up-regulated in tumor cells and during immortalization. Cell 90: 785-795, 1997.

7. Nakamura TM, Morin GB, Chapman KB, et al: Telomerase catalytic subunit homologs from fission yeast and human. Science 277: 955-959, 1997.

8. Weinrich SL, Pruzan R, Ma L, et al: Reconstitution of human telomerase with the template RNA component hTR and the catalytic protein subunit hTRT. Nat Genet 17: 498-502, 1997.

9. Bodnar AG, Ouellette M, Frolkis M, et al: Extension of lifespan by introduction of telomerase into normal human cells. Science 279: 349-352, 1998.

10. Kim NW, Piatyszek MA, Prowse KR, et al: Specific association of human telomerase activity with immortal cells and cancer. Science 266: 2011-2015, 1994.

11. Shay JW and Bacchetti S: A survey of telomerase activity in human cancer. Eur J Cancer 33: 787-791, 1997.

12. Lan KH, Kanai $\mathrm{F}$, Shiratori $\mathrm{Y}$, et al: In vivo selective gene expression and therapy mediated by adenoviral vectors for human carcinoembryonic antigen-producing gastric carcinoma. Cancer Res 57: 4279-4284, 1997.

13. Gu J, Kagawa S, Takakura M, Kyo S, Inoue M, Roth JA and Fang B: Tumor-specific transgene expression from the human telomerase reverse transcriptase promoter enables targeting of the therapeutic effects of the Bax gene to cancers. Cancer Res 60: 5359-5364, 2000.

14. Terazaki Y, Yano S, Yuge K, et al: An optimal therapeutic expression level is crucial for suicide gene therapy for hepatic metastatic cancer in mice. Hepatology 37: 155-163, 2003.

15. Koga S, Hirohata S, Kondo Y, et al: A novel telomerasespecific gene therapy: gene transfer of caspase- 8 utilizing the human telomerase catalytic subunit gene promoter. Hum Gene Ther 11: 1397-1406, 2000.

16. Majumdar AS, Hughes DE, Lichtsteiner SP, Wang Z, Lebkowski JS and Vasserot AP: The telomerase reverse transcriptase promoter drives efficacious tumor suicide gene therapy while preventing hepatotoxicity encountered with constitutive promoters. Gene Ther 8: 568-578, 2001.

17. Irving $\mathrm{J}$, Wang $\mathrm{Z}$, Powell $\mathrm{S}$, et al: Conditionally replicative adenovirus driven by the human telomerase promoter provides broad-spectrum antitumor activity without liver toxicity. Cancer Gene Ther 11: 174-185, 2004.

18. Zou W, Luo C, Zhang Z, et al: A novel oncolytic adenovirus targeting to telomerase activity in tumor cells with potent. Oncogene 23: 457-464, 2004.

19. Nagano S, Oshika H, Fujiwara H, Komiya S and Kosai K: An efficient construction of conditionally replicating adenoviruses that target tumor cells with multiple factors. Gene Ther 12: 1385-1393, 2005
20. Kamizono J, Nagano S, Murofushi Y, Komiya S, Fujiwara H, Matsuishi T and Kosai K: Survivin-responsive conditionally replicating adenovirus exhibits cancer-specific and efficient viral replication. Cancer Res 65: 5284-5291, 2005.

21. Komata T, Koga S, Hirohata S, et al: A novel treatment of human malignant gliomas in vitro and in vivo: FADD gene transfer under the control of the human telomerase reverse transcriptase gene promoter. Int J Oncol 19: 1015-1020, 2001.

22. Jacob D, Davis J, Zhu H, et al: Suppressing orthotopic pancreatic tumor growth with a fiber-modified adenovector expressing the TRAIL gene from the human telomerase reverse transcriptase promoter. Clin Cancer Res 10: 3535-3541, 2004.

23. Broccoli D, Young JW and De Lange T: Telomerase activity in normal and malignant hematopoietic cells. Proc Natl Acad Sci USA 92: 9082-9086, 1995.

24. Kolquist KA, Ellisen LW, Counter CM, et al: Expression of TERT in early premalignant lesions and a subset of cells in normal tissues. Nat Genet 19: 182-186, 1998.

25. Kyo S, Takakura M, Kohama T and Inoue M: Telomerase activity in human endometrium. Cancer Res 57: 610-614, 1997.

26. Masutomi K, Yu EY, Khurts S, et al: Telomerase maintains telomere structure in normal human cells. Cell 114: 241-253, 2003.

27. Ikoma T, Takahashi T, Nagano S, et al: A definitive role of RhoC in metastasis of orthotopic lung cancer in mice. Clin Cancer Res 10: 1192-1200, 2004.

28. Kawai T, Takahashi T, Esaki M, Ushikoshi H, Nagano S, Fujiwara $\mathrm{H}$ and Kosai K: Efficient cardiomyogenic differentiation of embryonic stem cell by fibroblast growth factor 2 and bone morphogenetic protein 2. Circ J 68: 691-702, 2004.

29. Yan P, Coindre JM, Benhattar J, Bosman FT and Guillou L: Telomerase activity and human telomerase reverse transcriptase mRNA expression in soft tissue tumors: correlation with grade, histology and proliferative activity. Cancer Res 59: 3166-3170, 1999.

30. Zhu X, Kumar R, Mandal M, et al: Cell cycle-dependent modulation of telomerase activity in tumor cells. Proc Natl Acad Sci USA 93: 6091-6095, 1996.

31. Takakura M, Kyo S, Kanaya T, Hirano H, Takeda J, Yutsudo M and Inoue M: Cloning of human telomerase catalytic subunit (hTERT) gene promoter and identification of proximal core promoter sequences essential for transcriptional activation in immortalized and cancer cells. Cancer Res 59: 551-557, 1999.

32. Ushikoshi H, Takahashi T, Chen X, et al: Local overexpression of HB-EGF exacerbates remodeling following myocardial infarction by activating noncardiomyocytes. Lab Invest 85 : 862-873, 2005.

33. Nagano S, Yuge K, Fukunaga M, Terazaki Y, Fujiwara H, Komiya $\mathrm{S}$ and Kosai K: Gene therapy eradicating distant disseminated micro-metastases by optimal cytokine expression in the primary lesion only: novel concepts for successful cytokine gene therapy. Int J Oncol 24: 549-558, 2004.

34. Yuge K, Takahashi T, Nagano S, et al: Adenoviral gene transduction of hepatocyte growth factor elicits inhibitory effects for hepatoma. Int J Oncol 27: 77-85, 2005.

35. Scheel C, Schaefer KL, Jauch A, et al: Alternative lengthening of telomeres is associated with chromosomal instability in osteosarcomas. Oncogene 20: 3835-3844, 2001.

36. Gan Y, Mo Y, Johnston J, Lu J, Wientjes MG and Au JL: Telomere maintenance in telomerase-positive human ovarian SKOV-3 cells cannot be retarded by complete inhibition of telomerase. FEBS Lett 527: 10-14, 2002.

37. Nakayama J, Tahara H, Tahara E, et al: Telomerase activation by hTRT in human normal fibroblasts and hepatocellular carcinomas. Nat Genet 18: 65-68, 1998.

38. Tomlinson RL, Ziegler TD, Supakorndej T, Terns RM and Terns MP: Cell cycle-regulated trafficking of human telomerase to telomeres. Mol Biol Cell 17: 955-965, 2006.

39. Chu RL, Post DE, Khuri FR and Van Meir EG: Use of replicating oncolytic adenoviruses in combination therapy for cancer. Clin Cancer Res 10: 5299-5312, 2004.

40. Ido A, Nakata K, Kato Y, et al: Gene therapy for hepatoma cells using a retrovirus vector carrying herpes simplex virus thymidine kinase gene under the control of human alpha-fetoprotein gene promoter. Cancer Res 55: 3105-3109, 1995.

41. Tanaka T, Kanai F, Okabe S, et al: Adenovirus-mediated prodrug gene therapy for carcinoembryonic antigen-producing human gastric carcinoma cells in vitro. Cancer Res 56: 1341-1345, 1996. 
42. Fukunaga M, Takamori S, Hayashi A, Shirouzu K and Kosai K: Adenoviral herpes simplex virus thymidine kinase gene therapy in an orthotopic lung cancer model. Ann Thorac Surg 73: 1740-1746, 2002.

43. Lanson NA Jr, Friedlander PL, Schwarzenberger P, Kolls JK and Wang G: Replication of an adenoviral vector controlled by the human telomerase reverse transcriptase promoter causes tumor-selective tumor lysis. Cancer Res 63: 7936-7941, 2003.
44. Painter RG, Lanson NA Jr, Jin Z, Park F and Wang G: Conditional expression of a suicide gene by the telomere reverse transcriptase promoter for potential post-therapeutic deletion of tumorigenesis. Cancer Sci 96: 607-613, 2005.

45. Fujimoto K, Kyo S, Takakura M, et al: Identification and characterization of negative regulatory elements of the human telomerase catalytic subunit (hTERT) gene promoter: possible role of MZF-2 in transcriptional repression of hTERT. Nucleic Acids Res 28: 2557-2562, 2000. 